\title{
On an extension of Knuth's rotation correspondence to reduced planar trees
}

\author{
Kurusch Ebrahimi-Fard and Dominique Manchon
}

\begin{abstract}
We present a bijection from planar reduced trees to planar rooted hypertrees, which extends Knuth's rotation correspondence between planar binary trees and planar rooted trees. The operadic counterpart of the new bijection is explained. Related to this, the space of planar reduced forests is endowed with a combinatorial Hopf algebra structure. The corresponding structure on the space of planar rooted hyperforests is also described.
\end{abstract}

Mathematics Subject Classification (2010). 05C05, 05C65, 16T05, $16 \mathrm{~T} 30$.

Keywords. Trees, hypertrees, rotation correspondence, operads, Hopf algebras.

\section{Introduction}

Rooted trees have been extensively used in many branches of pure and applied mathematics. Especially in the latter case they gained particular prominence due to the pioneering work on numerical integration methods by John Butcher in the 1960s [4], [15], [22]. He discovered a group structure in the context of Runge-Kutta integration methods. This group structure encodes the composition of so-called B-series. The latter are a generalization of Taylor series, in which rooted trees naturally appear, as Arthur Cayley noticed in his classical 1857 paper [7]. See [5], [23] for details. Since then, algebraic structures have become an important aspect in the study of numerical methods and related fields, see e.g. [2], [8], [24], [28], [37].

Somewhat after Butcher's seminal work, Gian-Carlo Rota and Saj-Nicole Joni observed in a seminal paper [29], that various combinatorial objects naturally possess compatible product and coproduct structures. With the work by William Schmitt [38] this ultimately converged into the notion of combinatorial Hopf algebra, i.e., as Marcelo Aguiar puts it, a connected graded vector space where the homogeneous components are spanned by finite sets of combinatorial objects, and the algebraic structures are given by particular constructions on those objects. Rooted trees provide a genuine example for such combinatorial objects, and several Hopf algebra structures have been described using them. In [21], [25], [26], [27], [41] the reader finds more details. In particular, Arne Dür, and later Christian Brouder [3] showed that the Butcher group identifies with the group of characters on the dual of a commutative 
graded Hopf algebra of rooted trees described by Alain Connes and Dirk Kreimer [14]. In [6] a combinatorial Hopf algebra structure on rooted trees was described that corresponds to the substitution law of B-series introduced in [12], see also [13]. In [36] a Hopf algebra on planar rooted trees was introduced in the context of LieButcher series on Lie groups.

Combinatorial Hopf algebras on rooted trees are generally related to the fact that free pre-Lie algebras are naturally described in terms of rooted trees [11], [16], [39]. In the case of Hans Munthe-Kaas and William Wright's noncocommutative Hopf algebra for Lie-Butcher series [36], this has been generalized to so-called $D$ algebras. Frédéric Chapoton observed in [9] that an operadic approach may provide an adequate perspective on the link between pre-Lie structures, the group of characters and combinatorial Hopf algebras.

The theory of correspondences between combinatorial objects is one of the main topics in combinatorics. As an example we mention Robinson's and Schensted's bijection between permutations and standard tableaux. Another example is Donald Knuth's rotation correspondence [30] for planar binary trees, which maps a planar binary tree with $n-1$ internal vertices into a planar rooted tree with $n$ vertices. A couple of years ago in a meeting in Lyon, J.-L. Loday suggested that it should be possible to extend this correspondence from planar binary trees (in which any vertex has two incoming edges and one outgoing edge) to planar reduced trees (in which any vertex has two incoming edges or more).

In this paper we solve this problem by generalizing Knuth's correspondence to a bijection between planar reduced trees and planar rooted hypertrees. This bijection is used to transfer a combinatorial Hopf algebra structure on planar reduced trees to planar rooted hypertrees. It turns out that the coproduct of the latter is very similar to the one in Munthe-Kaas and Wright's Hopf algebra. In a forthcoming article we will describe in more detail the underlying reason for this.

This paper is organized as follows. In Section 2 we introduce the notions of planar binary trees and planar rooted trees. The Butcher product on trees is presented. We recall Knuth's rotation correspondence between planar binary trees and planar rooted trees, which we then extend to a bijection from planar reduced trees to so-called planar rooted hypertrees. In Section 3 we briefly recall some notions from the theory of connected graded bialgebras, and then define a Hopf algebra on planar reduced trees respectively planar rooted hypertrees.

\section{Planar rooted trees and hypertrees}

Recall that a tree is an undirected connected graph made out of vertices and edges. It is without cycles, that is, any two vertices can be connected by exactly one simple path. We denote the set of vertices and edges of a tree $t$ by $V(t)$ and $E(t)$, respectively. In this section we introduce the objects of this work, which are particular classes of trees, i.e., planar binary (reduced) trees and planar rooted (hyper)trees. 
2.1. Planar trees. We start with the notion of a planar binary tree, which is a finite oriented tree given an embedding in the plane, such that all vertices have exactly two incoming edges and one outgoing edge. An edge can be internal (connecting two vertices) or external (with one loose end). The external incoming edges are the leaves. The root is the unique edge not ending in a vertex.
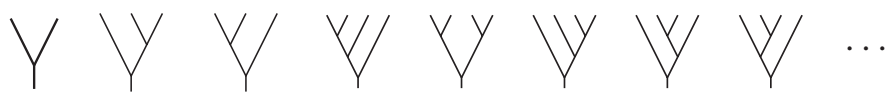

The single edge $\mid$ is the unique planar binary tree without internal vertices. We denote by $T_{\mathrm{pl}}^{\text {bin }}\left(\right.$ resp. $\left.\mathcal{T}_{\mathrm{pl}}^{\text {bin }}\right)$ the set (resp. the linear span) of planar binary trees. A simple grading for such trees is given in terms of the number of internal vertices. Alternatively, one can use the number of leaves. Observe that for any pair of planar binary trees $t_{1}, t_{2}$ we can build up a new planar binary tree via the grafting operation, $t_{3}:=t_{1} \vee t_{2}$, i.e., by considering the $Y$-shaped tree $Y$ (the unique planar binary tree with two leaves) and replacing the left branch (resp. the right branch) by $t_{1}$ (resp. $t_{2}$ ).

$$
|\vee|=Y \quad Y \vee|=Y \quad| \vee Y=Y \quad Y \vee Y=Y Y \mid \vee Y=Y
$$

Seen as a product on $\mathcal{T}_{\mathrm{pl}}^{\text {bin }}$, the grafting operation $\vee$ is neither associative nor commutative, $t_{1} \vee t_{2} \neq t_{2} \vee t_{1}$. In fact, $\left(\mathcal{T}_{\mathrm{pl}}^{\text {bin }}, \vee\right)$ is the free magmatic algebra generated by the tree $\mid$. Notice that this product is of degree one with respect to the grading in terms of internal vertices, i.e., for two trees $t_{1}, t_{2}$ of degrees $n_{1}, n_{2}$, respectively, the product $t_{1} \vee t_{2}$ is of degree $n_{1}+n_{2}+1$. However, with respect to the leave number grading this product is of degree zero.

A planar rooted tree is a finite oriented rooted tree given an embedding in the plane, such that all vertices, except one, have arbitrarily many incoming edges and one outgoing edge. The root is the one vertex without an outgoing edge.

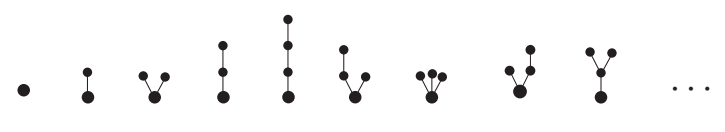

The single vertex $\bullet$ is the unique rooted tree without edges. Note that we put the root at the bottom of the tree. The set (resp. the linear span) of planar non-empty rooted trees will be denoted by $T_{\mathrm{pl}}\left(\mathrm{resp} . \mathcal{T}_{\mathrm{pl}}\right)$. A natural grading for such trees is given in terms of the number of edges. Another one is given by the number of vertices. Observe that any rooted tree of degree bigger than zero can be written in a unique way:

$$
t=B_{+}\left(t_{1} \cdots t_{n}\right),
$$

where $B_{+}$associates to the forest $t_{1} \cdots t_{n}$ the planar tree obtained by grafting all the 
planar trees $t_{j}, j=1, \ldots, n$, on a common root.

$$
B_{+}(\bullet)=\vdots \quad B_{+}(\bullet \bullet)=\because \quad B_{+}(\bullet \bullet)=\dot{\ddots} \quad B_{+}(\bullet \bullet)=\because .
$$

Recall that sometimes, one finds the notation $t=\left[t_{1} \cdots t_{n}\right]$ in the literature [3], [4]. Note that the order in which the branch trees are displayed has to be taken into account.

Further below we will recall the classical correspondence between these two types of trees, due to Knuth [30].

2.2. The Butcher product. Motivated by the use of (non-)planar rooted trees in the theory of numerical integration methods [4], [5], [21], [22], we introduce a planar version of the classical Butcher product. The (left) Butcher product of two planar rooted trees $t, u$ is defined by connecting the root of $t$ via a new edge to the root of $u$ such that $t$ becomes the leftmost branch tree, that is, for two trees $t=B_{+}\left(t_{1} \cdots t_{n}\right)$ and $u=B_{+}\left(u_{1} \cdots u_{p}\right)$ :

$$
t \circ \rightarrow u:=B_{+}\left(t u_{1} \cdots u_{p}\right)
$$

Observe that it is neither associative nor commutative, and, again contrarily to the non-planar case, it is also not NAP (Non-Associative Permutative) [31], i.e., it does not satisfy the identity $t \circ \rightarrow(u \circ \rightarrow v)=u \circ \rightarrow(t \circ \rightarrow v)$.

\subsection{Knuth's correspondence between planar binary and planar rooted trees.} Knuth describes in [30] a natural relation between planar rooted trees and planar binary trees, known as rotation correspondence. We only give a recursive definition of this bijection, and refrain from providing more details. The interested reader is referred to Marckert's paper [34] for a nice description of the rotational aspect.

Recall that by the single edge $\mid$ we denote the unique planar binary tree without internal vertices. Now we recursively define a map $\Phi: T_{\mathrm{pl}}^{\text {bin }} \rightarrow T_{\mathrm{pl}}$ by $\Phi(\mid):=\bullet$ and

$$
\Phi\left(t_{1} \vee t_{2}\right):=\Phi\left(t_{1}\right) \circ \rightarrow \Phi\left(t_{2}\right) .
$$

This map is clearly well defined and bijective, ${ }^{1}$ with its inverse recursively given by

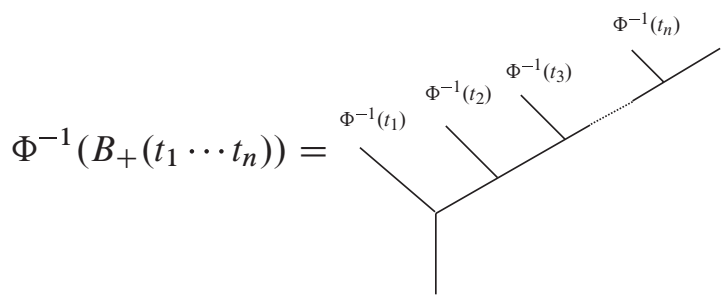

\footnotetext{
${ }^{1}$ It appears also in [20] in slightly different form.
} 
Here are the first few terms:

$$
\begin{gathered}
\Phi(\mid)=\bullet \quad \Phi(Y)=: \quad \Phi(Y)=\because \quad \Phi(Y)=\vdots \\
\Phi(Y)=\vdots \quad \Phi(Y)=\because \quad \Phi(Y)=\ddot{\bullet} \quad \Phi(Y)=\because \quad \Phi(Y)=\because .
\end{gathered}
$$

Note that this simple bijection implies that $\left(\mathcal{T}_{\mathrm{pl}}, \circ \rightarrow\right)$ is the free magmatic algebra generated by the one-vertex tree $\bullet$.

2.4. Reduced planar rooted trees and planar rooted hypertrees. A planar tree is called reduced if any inner vertex has at least two incoming edges. We denote by $T_{\mathrm{pl}}^{\text {red }}\left(\right.$ resp. $\left.\mathcal{T}_{\mathrm{pl}}^{\text {red }}\right)$ the set (resp. the linear span) of reduced planar trees. Any reduced planar tree can be described for $n>1$ as $t=\bigvee\left(t_{1}, \ldots, t_{n}\right)$, i.e., it can be obtained by considering the unique tree with one internal vertex and $n$ incoming edges, and replacing the $i^{\text {th }}$ branch by $t_{i}$. There is a partial order on $T_{\mathrm{pl}}^{\text {red }}$ defined as follows: $t_{1} \leq t_{2}$ if $t_{1}$ can be obtained from $t_{2}$ by glueing some inner vertices together. In particular, two comparable trees must have the same number of leaves. The minimal elements are the trees with only one inner vertex, and the maximal elements are the planar binary trees.

We would like to propose a way to extend the bijection $\Phi$, originally defined on planar binary trees, to reduced planar trees, thus answering the question raised by J.-L. Loday. The image of $T_{\mathrm{pl}}^{\text {red }}$ will be the space $H T_{\mathrm{pl}}$ of planar rooted hypertrees, which we introduce now.

Following C. Berge [1] (see also [10]), a hypergraph on a finite set $I$ of vertices is a nonempty set of parts of $I$ of cardinality at least 2 , which will be called the edges of the hypergraph. A path in a hypergraph is a sequence $i_{1}, \ldots, i_{k}$ of vertices such that any pair $\left\{i_{j}, i_{j+1}\right\}$ is included in an edge. A hypergraph is connected if any two vertices can be joined by a path. A hypertree [35], [10] is a connected hypergraph without cycles except those which are included in a single edge. Two different edges in a hypertree then meet at one single vertex or have empty intersection.

A rooted hypertree is a hypertree with a distinguished vertex. This defines a partial order on the set of edges as follows: $e<e^{\prime}$ if for any vertex $j$ in $e$ and any vertex $j^{\prime}$ in $e^{\prime}$ there is a path from the root to $j^{\prime}$ through $j$. This in turn defines a preorder on the vertices in an obvious way. For any edge $e^{\prime}$ not containing the root, there is a unique edge $e$ such that $e<e^{\prime}$ and $e \cap e^{\prime} \neq \emptyset$. The unique vertex in this intersection will be called the root of the edge $e^{\prime}$. Define a planar rooted hypertree as a rooted hypertree together with an embedding into the plane such that any edge is embedded in the boundary of a small topological disk. This defines a partial order on the vertices compatible with the preorder defined above, i.e., it determines a total order on each edge with the edge's root as minimal element, by running counterclockwise along 
the boundary of the disk. The following planar rooted hypertree

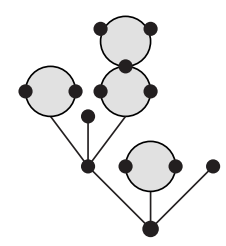

has seven edges altogether, three with 2 vertices, three with 3 vertices and one with 4 vertices. Each edge of cardinality bigger than 2 is represented by a blob. The vertices are drawn on the circle delimiting the blob, and are ordered counterclockwise starting from the edge's root.

There is a partial order on the set of all rooted planar hypertrees on a given set $I$ of vertices with root $r \in I$ fixed: $t_{1} \leq t_{2}$ if and only if any edge of $t_{2}$ is contained in an edge of $t_{1}$. The minimal element is the hypertree with only one edge equal to the whole $I$, and the maximal elements are planar rooted trees on $I$ with root $r$.

We are now ready to extend Knuth's rotation correspondence:

Theorem 1. The rotation correspondence from $T_{\mathrm{pl}}^{\mathrm{bin}}$ to $T_{\mathrm{pl}}$ extends to a bijection $\Phi: T_{\mathrm{pl}}^{\mathrm{red}} \rightarrow H T_{\mathrm{pl}}$, which, for any positive integer $p$, sends any planar reduced tree with $p$ leaves to a planar rooted hypertree with $p$ vertices.

Proof. For any ordered collection $\left(t_{1}, \ldots, t_{n}\right)$ of planar rooted hypertrees with respective roots $r_{j}$ we define $\beta\left(t_{1}, \ldots t_{n}\right)$ by collecting the roots $r_{j}$ into a common edge, in which the vertices are put in the reversed order. In particular, it implies that $r_{n}$ is the root of this new edge, hence the root of the new built hypertree. This certainly extends the Butcher product of two trees (1). We then extend $\Phi$ by setting recursively:

$$
\Phi\left(\bigvee\left(t_{1}, \ldots, t_{n}\right)\right):=\beta\left(\Phi\left(t_{1}\right), \ldots, \Phi\left(t_{n}\right)\right) .
$$

Any planar rooted hypertree can be written in a unique way as $\beta\left(s_{1}, \ldots, s_{n}\right)$, where $n$ is the cardinality of the leftmost edge containing the root. The inverse $\Phi^{-1}$ is then recursively defined as follows:

$$
\Phi^{-1}\left(\beta\left(s_{1}, \ldots, s_{n}\right)\right)=\bigvee\left(\Phi^{-1}\left(s_{1}\right), \ldots, \Phi^{-1}\left(s_{n}\right)\right) .
$$

Considering the example (2) above, we have

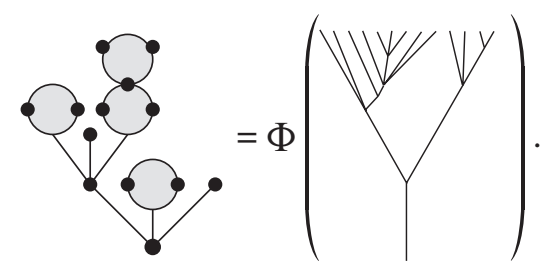


Recall that the reduced planar trees with $n$ leaves are in bijection with the cells of the $n-2$-associahedron. In particular, reduced planar trees with four leaves can be displayed on the pentagon like this:

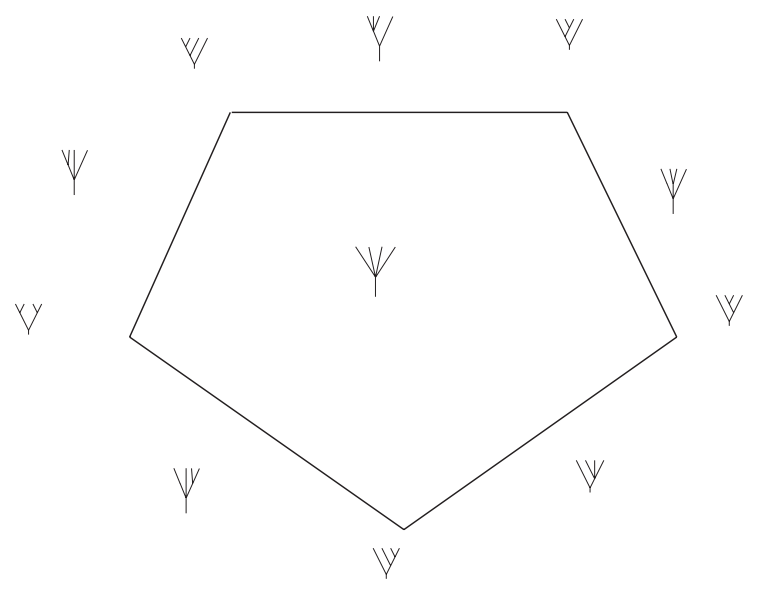

Under transformation $\Phi$ the picture transforms like this:

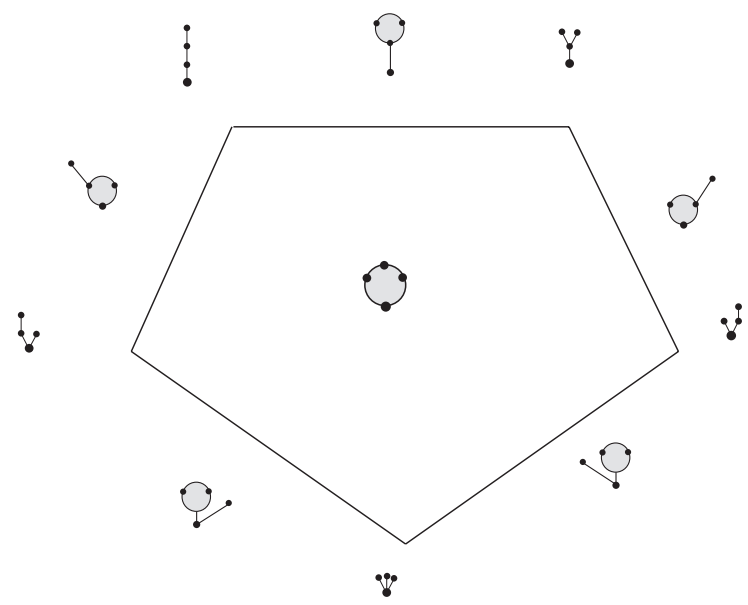

It is easy to show that $\Phi$ respects the partial orders defined above, which are two manifestations of the reverse incidence order of the associahedron.

2.5. Adding decorations. A planar binary tree decorated by a set $I$ is a planar binary tree together with a map $\delta$ form the set of its internal vertices to $I$. There are grafting operations $\vee_{i}, i \in I$, defined as in the undecorated case, except that the new internal vertex is decorated by $i$. This decoration procedure generalizes to planar reduced trees as follows: given a partitioned set $\mathcal{I}=I_{2} \amalg I_{3} \amalg \cdots$, a planar reduced tree decorated by $\mathcal{I}$ is a planar reduced tree together with a map $\delta$ from the set of its 
internal vertices to $\mathcal{I}$, which sends the internal vertices which have $n$ incoming edges into $I_{n}$. Any such decorated planar reduced rooted tree can be uniquely written in the form

$$
t=\bigvee_{i}\left(t_{1}, \ldots, t_{n}\right)
$$

with $i \in I_{n}$ for some $n \geq 2$, i.e., it can be obtained by considering the unique tree with one internal vertex decorated by $i$ and $n$ incoming edges, and replacing the $n^{\text {th }}$ branch by $t_{n}$.

Equations (3) and (4) also recursively define a bijection $\omega_{t}$ between the internal vertices of a reduced planar tree $t$ and the edges of the planar rooted hypertree $\Phi(t)$, which associates to any internal vertex of $t$ with $n$ incoming edges an edge of $\Phi(t)$ with $n$ vertices. The vertex of $t=\bigvee\left(t_{1}, \ldots, t_{n}\right)$ closest to the root (with $n$ incoming edges) is sent to the leftmost edge of $\Phi(t)$ containing the root. The bijection $\Phi$ hence gives rise to a bijection $\Phi_{\mathcal{I}}$ from $\mathcal{I}$-decorated reduced planar trees to rooted planar hypertrees with edges decorated by $\mathcal{I}$ (i.e., the edges with $n$ vertices are decorated by $I_{n}, n \geq 2$ ). The bijection $\Phi_{\mathcal{I}}$ is defined as follows:

$$
\Phi_{\mathcal{I}}(t, \delta)=\left(\Phi(t), \delta \circ \omega_{t}^{-1}\right) .
$$

Any such $\mathcal{I}$-decorated rooted planar hypertree can be uniquely written as:

$$
s=\beta_{i}\left(s_{1}, \ldots, s_{n}\right)
$$

with $i \in I_{n}$ for some $n \geq 2$, i.e., it can be obtained by collecting the roots of $s_{j}$, $j=1, \ldots, n$, this making the leftmost bottom edge, and decorating this new edge by $i$.

2.6. Operadic structure. Equation (4) recursively defines a bijection between the vertices of a planar rooted hypertree $s$ and the leaves of the reduced planar tree $\Phi^{-1}(s)$ (the root corresponding to the rightmost leaf). Any labeling of the vertices of $s$ thus corresponds to a labeling of the leaves of $\Phi^{-1}(s)$. On the example above this reads:

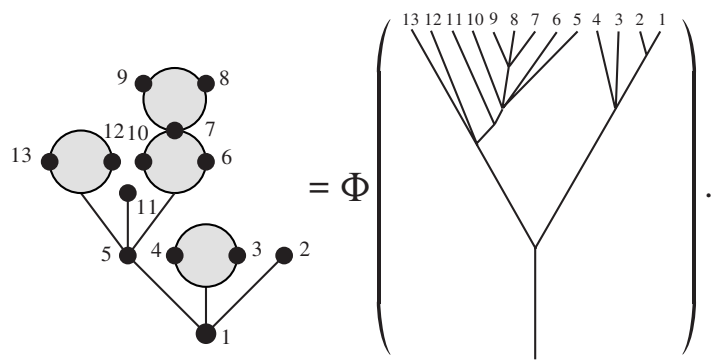

Recall that an $\mathbb{\$}$-object is a graded vector space $V=V_{1} \oplus V_{2} \oplus \cdots$ together with an action of the symmetric group $S_{k}$ on $V_{k}$ for any $k \geq 1$. For any partitioned set $\mathcal{I}=I_{2} \amalg I_{3} \amalg \cdots$ we consider the $\mathbb{\$}$-object $V(\mathcal{I})$ defined by $\left(V_{\mathcal{I}}\right)_{1}=k$ and 
$\left(V_{\mathcal{I}}\right)_{n}=k^{\left|I_{n}\right|} \otimes k\left[S_{n}\right]$ for $n \geq 2$. The vector space $\mathcal{T}_{\mathrm{pl}}^{\text {red, } \mathcal{I}}$ generated by $\mathcal{I}$-decorated reduced planar trees (see Section 2.5 above) naturally encodes the free operad on $V(\mathcal{I})$ : the partial composition $\sigma \circ_{i} \tau$ of two $\mathcal{I}$-decorated planar reduced trees with labeled leaves is obtained by replacing leaf number $i$ of $\sigma$ by $\tau$. The operadic structure of $\mathcal{I}$-decorated reduced planar rooted trees can be transferred to the linear span $H \widetilde{\mathcal{T}}_{\mathrm{pl}}$ of planar rooted hypertrees by means of $\Phi_{\mathcal{I}}^{-1}$ : the partial composition $t_{1} \circ_{i} t_{2}$ of two planar rooted hypertrees with labeled vertices is then obtained by replacing vertex number $i$ of hypertree $t_{1}$ by the root of the hypertree $t_{2}$, and putting the hypertree $t_{2}$ plugged this way on the right of the other edges stemming from vertex number $i$. This is easily seen when the vertex is the root of $t_{1}$, and the other vertices are treated by induction, by remarking that a vertex of $t_{1}$ different from the root is a vertex of the $j^{\text {th }}$ branch tree $t_{1, j}$ of $t_{1}$.

The fully transferred operadic structure on the vector space $H \mathcal{T}_{\mathrm{pl}}^{\mathcal{I}}$ of $\mathcal{I}$-decorated hypertrees is then the following: $\gamma\left(t ; t_{1}, \ldots, t_{n}\right)$ is given by replacing vertex number $i$ of $t$ by the root of $t_{i}$, and by putting the plugged hypertree $t_{i}$ on the right. This class of operads is known as generic magmatic operads [42].

\section{Hopf algebra structures on trees}

3.1. Connected filtered bialgebras. In general, $k$ denotes the ground field (of characteristic zero) over which all algebraic structures are defined. Recall the definition of a bialgebra, which is an algebra and coalgebra structure together with compatibility relations [29]. We denote a Hopf algebra by $\left(\mathscr{H}, m_{\mathscr{H}}, \eta_{\mathscr{H}}, \Delta_{\mathscr{H}}, \epsilon \mathscr{H}, S\right)$. It is a bialgebra together with a particular $k$-linear map, i.e., the antipode $S: \mathscr{H} \rightarrow \mathscr{H}$, satisfying the Hopf algebra axioms [40], [33]. In the following we omit subscripts if there is no danger of confusion. We denote the unit by $\mathbf{1}=\eta_{\mathscr{H}}(1)$. Let $\mathscr{H}$ be a connected filtered bialgebra, that is,

$$
k=\mathscr{H}^{(0)} \subset \mathscr{H}^{(1)} \subset \cdots \subset \mathscr{H}^{(n)} \subset \cdots, \bigcup_{n \geq 0} \mathscr{H}^{(n)}=\mathscr{H} .
$$

For any $x \in \mathscr{H}^{(n)}$ we have, using a variant of Sweedler's notation [40],

$$
\Delta(x)=x \otimes \mathbf{1}+\mathbf{1} \otimes x+\sum_{(x)} x^{\prime} \otimes x^{\prime \prime},
$$

where the filtration degrees of $x^{\prime}$ and $x^{\prime \prime}$ are strictly smaller than $n$. Recall that by definition we call an element $x \in \mathscr{H}$ primitive if

$$
\bar{\Delta}(x):=\Delta(x)-x \otimes \mathbf{1}-\mathbf{1} \otimes x=0 .
$$

The antipode $S: \mathscr{H} \rightarrow \mathscr{H}$ is defined in terms of the equations

$$
S * \mathrm{Id}=m \circ(S \otimes \mathrm{Id}) \circ \Delta=\eta \circ \epsilon=\mathrm{Id} * S,
$$


where the convolution product for two linear maps $f, g \in \mathscr{L}(\mathscr{H}, \mathcal{H})$ is defined by $f * g:=m \circ(f \otimes g) \circ \Delta: \mathscr{H} \rightarrow \mathcal{H}$, i.e.,

$$
(f * g)(x)=f(x) g(\mathbf{1})+f(\mathbf{1}) g(x)+\sum_{(x)} f\left(x^{\prime}\right) g\left(x^{\prime \prime}\right) \in \mathscr{H} .
$$

It yields an associative algebra with unit $e:=\epsilon$ on the vector space $\mathscr{L}(\mathscr{H}, \mathscr{H})$. The antipode always exists for connected filtered bialgebras, hence any connected filtered bialgebra is a connected filtered Hopf algebra. Equations (5) imply the following recursive formulas for the antipode starting with $S(\mathbf{1})=\mathbf{1}$ and for $x \in \operatorname{ker} \epsilon$ :

$$
S(x)=-x-\sum_{(x)} S\left(x^{\prime}\right) x^{\prime \prime}=-x-\sum_{(x)} x^{\prime} S\left(x^{\prime \prime}\right) .
$$

Let $\mathscr{H}$ be a graded Hopf algebra. The grading induces a biderivation $Y: \mathscr{H}^{(n)} \rightarrow$ $\mathscr{H}^{(n)}$ defined on homogeneous elements by $x \longmapsto n x$.

3.2. The Butcher-Connes-Kreimer Hopf algebra of rooted forests. An important example of a connected filtered, in fact, graded, Hopf algebra is given by the Butcher-Connes-Kreimer Hopf algebra $\mathscr{H}_{\mathrm{BCK}}$ of rooted forests over $k$, graded by the number of vertices [4], [14], [15], [33]. It is the free unital commutative algebra on the linear space $\mathcal{T}$ spanned by nonempty non-planar rooted trees. We list all rooted trees up to degree 5 :

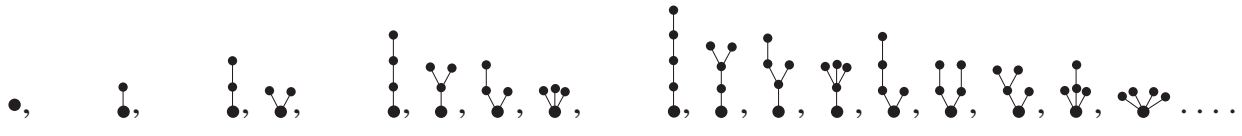

The empty set is denoted $\mathbf{1}$, and is the unit. A rooted forest is a finite collection $s=\left(t_{1}, \ldots, t_{n}\right)$ of rooted trees, which we simply denote by the (commutative) product $t_{1} \cdots t_{n}$. Recall that the operator $B_{+}$associates to the forest $s$ the tree $B_{+}(s)$ obtained by grafting the connected components $t_{j}$ on a common new root. $B_{+}(\mathbf{1})$ is the unique rooted tree $\bullet$ with only one vertex. The Butcher-Connes-Kreimer coproduct on a rooted tree $t$ is described in terms of admissible cuts as follows:

$$
\Delta_{\mathrm{BCK}}(t)=t \otimes \mathbf{1}+\mathbf{1} \otimes t+\sum_{c \in \operatorname{Adm}(t)} P^{c}(t) \otimes R^{c}(t) .
$$

Here $\operatorname{Adm}(t)$ is understood as the set of admissible cuts of a tree, i.e., the set of collections of edges such that any path from the root to a leaf contains at most one edge of the collection. ${ }^{2}$ We denote by $P^{c}(t)$ (resp. $R^{c}(t)$ ) the pruning (resp. the trunk) of $t$, i.e., the subforest formed by the edges above the cut $c \in \operatorname{Adm}(t)$ (resp. the subforest formed by the edges under the cut). Note that the trunk of a tree is a

\footnotetext{
${ }^{2}$ In order to make this picture completely correct, we must stress that for any nonempty tree two admissible cuts must be associated with the empty collection: the empty cut and the total cut.
} 
tree, but the pruning of a tree may be a forest. An elementary cut is a cut of only one edge. See [25], [26], [27], [33] for more details on the combinatorics of rooted trees and Hopf algebras.

3.3. Groups associated with augmented operads. Following [9], we introduce an augmented operad, which is an operad $\mathcal{P}$ such that $\operatorname{dim} \mathcal{P}_{0}=0$ and $\operatorname{dim} \mathcal{P}_{1}=1$, i.e., such that there is no 0 -ary operation and that the only 1 -ary operation is the unit $e$. The group $G_{\mathcal{P}}$ is defined in [9] as the group of invertible elements in the product

$$
\prod_{n \geq 1}\left(\mathcal{P}_{n}\right)_{S_{n}}
$$

which is the completed free $\mathcal{P}$-algebra with one generator. An element in this product can be written as a (possibly infinite) sum $g=\sum_{n \geq 1} g_{n}$ with $g_{n} \in\left(\mathscr{P}_{n}\right)_{S_{n}}$. It is invertible if and only if its first component $g_{1}$ is nonzero. We will consider a slightly smaller group:

$$
G_{\mathcal{P}}^{e}:=\left\{g=\sum_{n \geq 1} g_{n}, g_{1}=e\right\} .
$$

The advantage of this definition is the pro-nilpotency property. The associated Lie algebra is given by

$$
\mathfrak{g}_{\mathcal{P}}^{e}:=\left\{x=\sum_{n \geq 2} x_{n}\right\},
$$

with the Lie bracket given by

$$
[x, y]:=x \curvearrowleft y-y \curvearrowleft x,
$$

where the bilinear operation $\curvearrowleft$ is defined as follows: for any $x_{m} \in\left(\mathscr{P}_{m}\right)_{S_{m}}$ and $y_{n} \in\left(\mathcal{P}_{n}\right)_{S_{n}}$, with representatives $X_{m} \in \mathcal{P}_{m}$ and $Y_{n} \in \mathcal{P}_{n}$ respectively,

$$
x_{m} \curvearrowleft y_{n}:=\overline{\sum_{i=1}^{m} X_{m} \circ_{i} Y_{n}}=\overline{\sum_{i=1}^{m} \gamma(X_{m} ; \underbrace{e, \ldots, e}_{i-1 \text { terms }}, Y_{n}, e, \ldots, e)} .
$$

Here the bars stand for taking the class modulo the right action of $S_{m+n}$. This is well defined by virtue of the equivariance axioms for an operad. The operation $\curvearrowleft$ defined above is right pre-Lie [11], i.e., we have

$$
(x \curvearrowleft y) \curvearrowleft z-x \curvearrowleft(y \curvearrowleft z)=(x \curvearrowleft z) \curvearrowleft y-x \curvearrowleft(z \curvearrowleft y) .
$$

Of course, we also could consider the left pre-Lie operation $\curvearrowright$ defined by $x \curvearrowright y:=$ $y \curvearrowleft x$, subject to the left pre-Lie relation

$$
(x \curvearrowright y) \curvearrowright z-x \curvearrowright(y \curvearrowright z)=(y \curvearrowright x) \curvearrowright z-y \curvearrowright(x \curvearrowright z) .
$$

The reader immediately verifies that $[x, y]=x \curvearrowleft y-y \curvearrowleft x=-(x \curvearrowright y-y \curvearrowright x)$. 
3.4. A Hopf algebra structure on reduced planar forests. We now define a graded connected Hopf algebra structure on planar reduced forests, with grading given by the total number of inner vertices. First, we extend $\mathcal{T}_{\mathrm{pl}}^{\text {red }}$ to the free noncommutative algebra of reduced planar rooted forests, denoted by $\mathscr{H}_{\mathrm{pl}}^{\text {red }}$, with the one-edge tree $\mid$ as unit and the multiplication given by concatenation. We define a coproduct on reduced planar trees in terms of admissible cuts of a tree $t \in \mathcal{T}_{\mathrm{pl}}^{\text {red }}$, i.e., a (possibly empty) subset $c$ of edges not connected to a leaf with the rule that along any path from the root of $t$ to any of its leaves there is at most one edge in $c$. The edges in $c$ are naturally ordered from left to right. To any admissible cut $c$ always corresponds then a unique subforest $P^{c}(t)$, the pruning, obtained by concatenation of the subtrees obtained by cutting the edges in $c$, in the order defined as above. Then we define the coproduct

$$
\Delta_{2}(t)=\sum_{c \in \operatorname{Adm} t} P^{c}(t) \otimes R^{c}(t),
$$

where $R^{c}(t)$ is the trunk, obtained by replacing each subtree of $P^{c}(t)$ with a single leaf. Note that the trunk of a tree is a tree, but the pruning of a tree may be a forest. We present a few examples:

$$
\begin{aligned}
& \Delta_{2}(Y)=Y \otimes|+| \otimes Y \\
& \Delta_{2}(Y)=Y \otimes|+| \otimes Y+Y \otimes Y \\
& \Delta_{2}(Y)=Y \otimes|+| \otimes Y+Y \otimes Y \\
& \Delta_{2}(Y)=Y Y \otimes|+| \otimes Y Y+Y \otimes Y+Y \otimes Y \\
& \Delta_{2}(Y Y)=Y Y \otimes|+| \otimes Y Y+Y \otimes Y+Y \otimes Y+Y Y \otimes Y \\
& \Delta_{2}(Y)=Y / \otimes|+| \otimes Y+Y \otimes Y+Y \otimes Y \\
& \Delta_{2}(Y)=Y \otimes|+| \otimes Y+Y \otimes Y+Y \otimes Y \\
& \Delta_{2}(Y)=Y \otimes|+| \otimes Y+Y \otimes Y+Y \otimes Y \\
& \Delta_{2}(Y)=Y \otimes|+| \otimes Y+Y \otimes Y
\end{aligned}
$$

Two compatible gradings can be chosen: the number of inner vertices, or alternatively the number of leaves minus the number of trees in the forest. We remark here that several Hopf subalgebras are readily identified. First, the binary forests obviously form a Hopf subalgebra $\mathscr{H}_{\mathrm{pl}}^{\mathrm{bin}}$ of $\mathscr{H}_{\mathrm{pl}}^{\text {red }}$, which in turn contains two other Hopf subalgebras, i.e., the Hopf subalgebra $\mathscr{H}_{\mathrm{r}, \mathrm{pl}}^{\text {bi }} \subset \mathscr{H}_{\mathrm{pl}}^{\text {bin }}$ (resp. $\mathscr{H}_{\mathrm{l}, \mathrm{pl}}^{\mathrm{bin}} \subset \mathscr{H}_{\mathrm{pl}}^{\text {bi }}$ ) of right-(resp. left)-combed 
binary planar rooted forests, generated by the trees $t_{\mathrm{r}}^{(n)}\left(\operatorname{resp} . t_{1}^{(n)}\right)$ recursively defined by $t_{\mathrm{r}}^{(1)}:=\mid=: t_{1}^{(1)}$ and $t_{\mathrm{r}}^{(n)}:=\mid \vee t_{\mathrm{r}}^{(n-1)}$, (resp. $\left.t_{1}^{(n)}:=t_{1}^{(n-1)} \vee \mid\right), n>1$. Also, observe that the trees with only one inner vertex, let us call them reduced corollas, are all primitive.

Remark that the two gradings coincide on the Hopf subalgebra $\mathscr{H}_{\mathrm{pl}}^{\text {bin }}$. It is immediate to adapt this construction to the decorated setting described in Section 2.5. Details are left to the reader.

Remark 2. The definition of this Hopf algebra is very similar to the definition of L. Foissy's Hopf algebra $\mathscr{H}_{\mathrm{F}}$ of planar rooted trees [19], and one could believe that $\mathscr{H}_{\mathrm{pl}}^{\text {red }}$ is a graded Hopf subalgebra of it: this is not the case. Indeed the dimension of the degree 2 component of $\mathscr{H}_{\mathrm{pl}}^{\text {red }}$ is 3 for the first grading, a basis being ( $Y, Y, Y Y$ ) and 4 for the second grading (add $Y$ to the basis), whereas the degree 2 component of $\mathscr{H}_{\mathrm{F}}$ is 2-dimensional, generated by $\bullet$ and $\bullet \bullet$.

3.5. The associated pre-Lie structure. Let $\left(\mathscr{H}_{\mathrm{pl}}^{\text {red }}\right)^{\circ}$ be the graded dual of $\mathscr{H}_{\mathrm{pl}}^{\text {red }}$. We consider the normalized dual basis $\left(\delta_{t}^{\prime}\right)$ of the basis of forests, defined by

$$
\left\langle\delta_{t_{1} \cdots t_{k}}^{\prime}, t_{1} \cdots t_{k}\right\rangle=\sigma\left(t_{1}\right) \cdots \sigma\left(t_{k}\right),
$$

where $\sigma\left(t_{j}\right)$ is the symmetry factor of the tree $t_{j}$, and $\left\langle\delta_{t_{1} \cdots t_{k}}^{\prime}, s\right\rangle=0$ if $s$ is a forest different from $t_{1} \cdots t_{k}$. The correspondence $t_{1} \cdots t_{k} \mapsto \delta_{t_{1} \cdots t_{k}}^{\prime}$ yields a linear isomorphism $\delta^{\prime}: \mathscr{H}_{\mathrm{pl}}^{\text {red }} \rightarrow\left(\mathscr{H}_{\mathrm{pl}}^{\text {red }}\right)^{\circ}$. If $t$ and $u$ are planar reduced trees, $\delta_{t}^{\prime}$ and $\delta_{u}^{\prime}$ are infinitesimal characters of the Hopf algebra $\mathscr{H}_{\mathrm{pl}}^{\text {red }}$, hence so is the Lie bracket defined in terms of the convolution product, $\left[\delta_{t}^{\prime}, \delta_{u}^{\prime}\right]=\delta_{t}^{\prime} \star_{2} \delta_{u}^{\prime}-\delta_{u}^{\prime} \star_{2} \delta_{t}^{\prime}$. Recall that an infinitesimal character maps the one-edge tree $\mid$ as well as any forest $t_{1} \cdots t_{k}, k>1$, to zero. The definition of the convolution product yields

$$
\left[\delta_{t}^{\prime}, \delta_{u}^{\prime}\right]=\delta_{t \curvearrowright \sigma u-u \curvearrowright \sigma}^{\prime},
$$

where we define, for any reduced planar rooted tree $t$,

$$
t \curvearrowright_{\sigma} u=\sum_{v \in T_{\mathrm{pl}}^{\mathrm{red}}} \frac{\sigma(t) \sigma(u)}{\sigma(v)} N(t, u, v) v .
$$

The coefficient $N(t, u, v)$ is the number of elementary cuts $c$ of the tree $v$ (in the sense of the previous subsection) such that $P^{c}(v)=t$ and $R^{c}(v)=u$. The coefficient

$$
M(t, u, v):=\frac{\sigma(t) \sigma(u)}{\sigma(v)} N(t, u, v)
$$

is the number of ways to graft $t$ on a leaf of the tree $u$ in order to obtain the tree $v$. Hence $t \curvearrowright \sigma u$ is the sum of all the possible graftings of $t$ on $u$. The left pre-Lie 
relation holds:

$$
s \curvearrowright_{\sigma}(t \curvearrowright \sigma u)-\left(s \curvearrowright_{\sigma} t\right) \curvearrowright_{\sigma} u=t \curvearrowright_{\sigma}\left(s \curvearrowright_{\sigma} u\right)-(t \curvearrowright \sigma s) \curvearrowright \sigma u .
$$

Indeed, both sides are expressed as the sum of all possible ways of grafting $s$ and $t$ on two different leaves of $u$. This pre-Lie structure, more precisely the associated right pre-Lie structure $\curvearrowleft_{\sigma}$, is a particular example of pre-Lie structures described in Section 3.3, where the corresponding operad is the free operad generated by one operation in each arity $\geq 2$. The associated Lie algebra structure on $\mathcal{T}_{\mathrm{pl}}^{\text {red }}$ is defined by $[t, u]:=t \curvearrowright_{\sigma} u-u \curvearrowright_{\sigma} t$, and gives rise to the Lie algebra of the (pro-nilpotent) group of characters, that is, multiplicative maps on the Hopf algebra $\mathscr{H}_{\mathrm{pl}}^{\text {red }}$, which identifies with the group $G_{\mathscr{F}(V)}^{e, \text { op }}$ associated with the free operad $\mathcal{F}(V)$ on the $\mathbb{D}$ object $V=\left(k\left[S_{n}\right]\right)_{n \geq 1}$, but with multiplication reversed. ${ }^{3}$ Let us remark that the commutative Hopf algebra, which follows via the Cartier-Milnor-Moore theorem from the group $G_{\mathscr{F}(V)}^{e, \text { op }}$, is not isomorphic to $\mathscr{H}_{\mathrm{pl}}^{\text {red }}$, but is just a quotient.

The same construction with planar binary trees yields the group of characters of the Hopf algebra $\mathscr{H}_{\mathrm{pl}}^{\text {bin }}$, which identifies with the group $G_{\mathscr{B}}^{e, \text { op }}$ associated with the free binary operad (with multiplication reversed). The free binary operad is the free operad $\mathcal{F}(W)$ on the $\$$-object $W$ such that $W_{1}=k, W_{2}=k\left[S_{2}\right]$ and $W_{n}=\{0\}$ for $n \geq 3$. Finally the same construction with $\mathcal{I}$-decorated trees (with the notations of Section 2.5) yields the group $G_{\mathscr{F}\left(V_{\mathcal{I}}\right)}^{e, \text { op }}$, where $\mathcal{F}\left(V_{\mathcal{I}}\right)$ is the free operad on the $\$$-object $V_{\mathcal{I}}$ defined by $\left(V_{\mathcal{I}}\right)_{1}=k$ and $\left(V_{\mathcal{I}}\right)_{n}=k^{\left|I_{n}\right|} \otimes k\left[S_{n}\right]$ for $n \geq 2$.

3.6. A Hopf algebra structure on planar rooted hyperforests. We extend the linear isomorphism $\Phi: \mathcal{T}_{\mathrm{pl}}^{\text {red }} \rightarrow H \mathcal{T}_{\mathrm{pl}}$ to a graded algebra isomorphism still denoted by $\Phi: \mathscr{H}_{\mathrm{pl}}^{\text {red }} \rightarrow H \mathscr{H}_{\mathrm{pl}}$, where $H \mathscr{H}_{\mathrm{pl}}$ stands for the free noncommutative algebra of rooted planar hyperforests. The grading is given by the total number of edges. The Hopf algebra structure on $\mathscr{H}_{\mathrm{pl}}^{\text {red }}$ can be transferred on $H \mathscr{H}_{\mathrm{pl}}$ by $\Phi$. The coproduct $\Delta=(\Phi \otimes \Phi) \circ \Delta_{2} \circ \Phi^{-1}$ can then be made explicit as follows.

We introduce the concept of right admissible cut in the spirit of Munthe-Kaas and Wright [36]. For any vertex $v \in V(t)$ we denote by $\mathrm{f}(v)$ its fertility, i.e., the number of edges with root $v$. Recall that we work with planar hypertrees. Hence, we may enumerate the incoming edges of each vertex $v \in V(t)$ counterclockwise from 1 to $\mathrm{f}(v)$. For any vertex $v$ and for any $i \in\{1, \ldots, \mathrm{f}(v)\}$ the $i^{\text {th }}$ single right vertex-cut associated to $v$ is the subset $c_{v}^{(i)} \subset E(t)$ of the $i$ first edges with root $v$ with respect to the order above. To each single right vertex-cut $c_{v}^{(i)}$ we may associate a sub-hypertree $P^{c_{v}^{(i)}}(t)$ obtained from $t$ by removing the edges $c_{v}^{(i)}(t)$ from the vertex $v$ in $t$ and grafting them to a new root resulting in a single planar rooted hypertree. We denote by $R^{c_{v}^{(i)}}(t)$ the remaining tree. A right vertex-cut $C$ is a (possibly empty) collection of single right vertex-cuts. A (right) vertex-cut $C$ is called admissible if any path

\footnotetext{
${ }^{3}$ This is due to the fact that the Lie algebra structure comes from a left pre-Lie operation.
} 
from the root to any vertex of $t$ encounters at most one single right vertex-cut. The single vertex-cuts in an admissible $C$ are naturally ordered from left to right, thus giving rise to a planar hyperforest $P^{C}(t)$. We denote by $R^{C}(t)$ the remaining tree. By $\operatorname{RAdm}(t)$ we denote the set of admissible right vertex-cuts. We define in terms of admissible (right) vertex-cuts the following coproduct:

$$
\Delta(t)=\sum_{C \in \operatorname{RAdm}(t)} P^{C}(t) \otimes R^{C}(t),
$$

We list a few coproducts below. Observe the conservation of the number of edges.

$$
\begin{aligned}
& \Delta(\bullet)=\bullet \otimes \bullet, \quad \Delta(\bullet)=: \otimes \bullet+\bullet \otimes:
\end{aligned}
$$

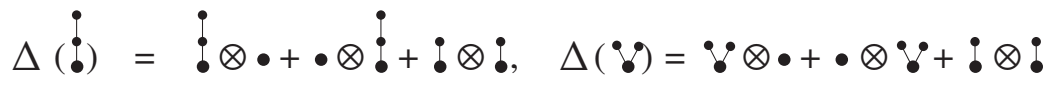

$$
\begin{aligned}
& \Delta\left(\begin{array}{l}
\vdots \\
\Delta
\end{array}\right)
\end{aligned}
$$

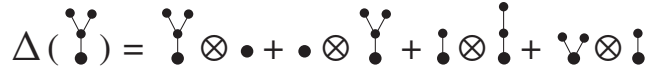

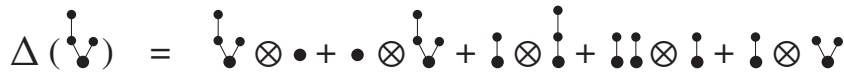

$$
\begin{aligned}
& \Delta(\because)=\because \otimes \bullet+-\otimes \because+\dot{0} \otimes \ddot{\bullet}+\dot{i} \otimes \\
& \Delta(\ddot{\bullet})=\ddot{\bullet} \otimes \bullet+\bullet \otimes \ddot{\bullet}+: \otimes \ddot{\bullet}+\because \otimes:
\end{aligned}
$$

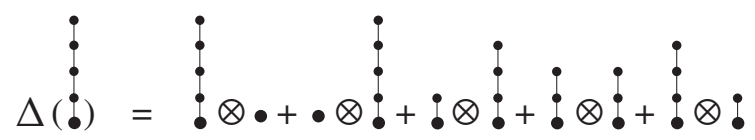

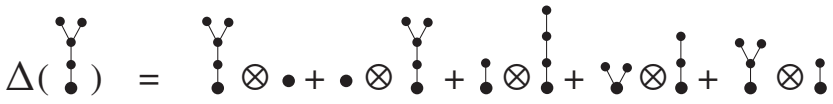

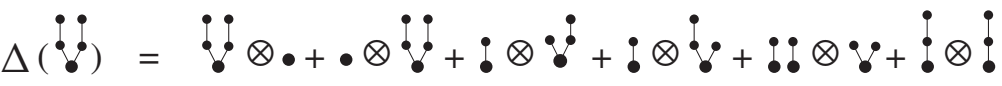

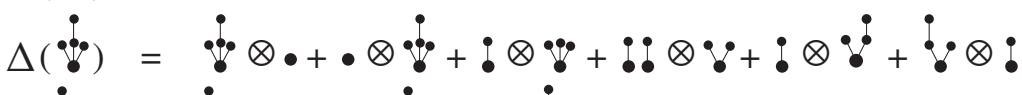

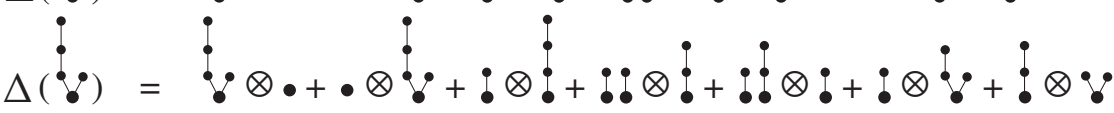

$$
\begin{aligned}
& \Delta\left(\vartheta_{)}=\varphi_{\otimes}+\bullet \otimes \varphi_{+} \otimes:\right.
\end{aligned}
$$

We note that via the bijection $\Phi$ we identify the Hopf subalgebras $\Phi\left(\mathscr{H}_{\mathrm{pl}}^{\text {bin }}\right)=$ $\mathscr{H}_{\mathrm{pl}} \subset H \mathscr{H}_{\mathrm{pl}}$ and $\Phi\left(\mathscr{H}_{l, p l}^{\text {bin }}\right)=\mathscr{H}_{\mathrm{pl}}^{\text {lad }} \subset \mathscr{H}_{\mathrm{pl}}$ and $\Phi\left(\mathscr{H}_{\mathrm{r}, \mathrm{pl}}^{\text {bi }}\right)=\mathscr{H}_{\mathrm{pl}}^{\text {cor }} \subset \mathscr{H}_{\mathrm{pl}}$ of ladder trees and corollas, respectively. Reduced corollas with $n$ leaves are mapped to blobs with $n$ vertices drawn on the circle delimiting the blob. 
This Hopf algebra structure is related to the pre-Lie structure $\rightarrow$ defined on $H \mathcal{T}_{\text {pl }}$ by

$$
s_{1} \rightarrow s_{2}:=\Phi\left(\Phi^{-1}\left(s_{1}\right) \curvearrowright_{\sigma} \Phi^{-1}\left(s_{2}\right)\right),
$$

where $\curvearrowright_{\sigma}$ is the pre-Lie product defined in Subsection 3.5. The associated Lie algebra is of course isomorphic to the one defined in same subsection. It is then another presentation of the opposite Lie algebra of the pro-nilpotent group $G_{\mathscr{F}(V)}^{e}$ associated with the free operad on the $\$$-object $V$ defined in Section 3.5. The same construction with planar rooted trees gives back the group $G_{\mathscr{B}}^{e}$ associated with the free binary operad (modulo reversing the multiplication or, what is the same, changing the sign of the Lie bracket). The same construction holds for $\mathcal{I}$-decorated hypertrees, leading to another presentation of the opposite Lie algebra of the pro-nilpotent group $G_{\mathcal{F}_{V(\mathcal{I})}}^{e}$ associated with the free operad on the $\$$-object $V(\mathcal{I})$ : details are left to the reader.

Acknowledgements. We would like to thank F. Chapoton for very helpful discussions. We thank H. Munthe-Kaas and A. Lundervold for comments. The first author is supported by a Ramón y Cajal research grant from the Spanish government, as well as the project MTM2011-23050 of the Ministerio de Economía y Competitividad. We thank the CNRS (GDR Renormalisation) and Agence Nationale de la Recherche (projet CARMA) for support.

\section{References}

[1] C. Berge, Hypergraphes. North-Holland Math. Library 43. North-Holland, Amsterdam 1989. Zbl 0674.05001 MR 1013569

[2] H. Berland and B. Owren, Algebraic structures on ordered rooted trees and their significance to Lie group integrators. In Group theory and numerical analysis, CRM Proc. Lecture Notes 39, Amer. Math. Soc., Providence, RI, 2005, 49-63. Zbl 1080.65056 MR 2182810

[3] Ch. Brouder, Runge-Kutta methods and renormalization. Europ. Phys. J. C 12 (2000), 512-534.

[4] J. C. Butcher, An algebraic theory of integration methods. Math. Comp. 26 (1972), 79-106. Zbl 0258.65070 MR 0305608

[5] J. C. Butcher, The numerical analysis of ordinary differential equations. John Wiley \& Sons Ltd., Chichester 1987. Zbl 0616.65072 MR 878564

[6] D. Calaque, K. Ebrahimi-Fard, and D. Manchon, Two interacting Hopf algebras of trees: A Hopf-algebraic approach to composition and substitution of B-series. Adv. in Appl. Math. 47 (2011), 282-308. Zbl 1235.16032 MR 2803804

[7] A. Cayley, On the theory of analytical forms called trees. Phil. Mag. 13 (1857), 172-176.

[8] E. Celledoni, R. I. McLachlan, B. Owren, G. R. W. Quispel, Energy-preserving integrators and the structure of B-series. Found. Comput. Math. 10 (2010), 673-693. Zbl 1205.65325 MR 2728426 
[9] F. Chapoton, Rooted trees and an exponential-like series. Preprint 2002. arXiv:math/0209104

[10] F. Chapoton, Hyperarbres, arbres enracinés et partitions pointées. Homology Homotopy Appl. 9 (2007), 193-212. Zbl 1109.05107 MR 2280292

[11] F. Chapoton and M. Livernet, Pre-Lie algebras and the rooted trees operad. Internat. Math. Res. Notices 2001 (2001), 395-408. Zbl 1053.17001 MR 1827084

[12] Ph. Chartier, E. Hairer, and G. Vilmart, A substitution law for B-series vector fields. Preprint INRIA No. 5498, Rennes 2005. hal.inria.fr/inria-00070509/PS/RR-5498.ps

[13] Ph. Chartier, E. Hairer, and G. Vilmart, Algebraic structures of B-series. Found. Comput. Math. 10 (2010), 407-427. Zbl 1201.65124 MR 2657947

[14] A. Connes and D. Kreimer, Hopf algebras, renormalization and noncommutative geometry. Comm. Math. Phys. 199 (1998), 203-242. Zbl 0932.16038 MR 1660199

[15] A. Dür, Möbius functions, incidence algebras and power series representations. Lecture Notes in Math. 1202, Springer-Verlag, Berlin 1986. Zbl 0592.05006 MR 857100

[16] A. Dzhumadil'daev and C. Löfwall, Trees, free right-symmetric algebras, free Novikov algebras and identities. Homology Homotopy Appl. 4 (2002), no. 2, 165-190. Zbl 1029.17001 MR 1918188

[17] K. Ebrahimi-Fard and D. Manchon, A Magnus- and Fer-type formula in dendriform algebras. Found. Comput. Math. 9 (2009), 295-316. Zbl 1173.17002 MR 2496553

[18] K. Ebrahimi-Fard and D. Manchon, Dendriform equations. J. Algebra 322 (2009), 40534079. Zbl 1229.17001 MR 2556138

[19] L. Foissy, Les algèbres de Hopf des arbres enracinés décorés, I, II. Bull. Sci. Math. 126 (2002), 193-239; ibid. 249-288. Zbl 1013.16026 MR 1905177 Zbl 1013.16027 MR 1909461

[20] L. Foissy, The infinitesimal Hopf algebra and the poset of planar forests. J. Algebraic Combin. 30 (2009), 277-309. Zbl 1192.16033 MR 2545498

[21] R. Grossman and R. G. Larson, Hopf-algebraic structure of families of trees. J. Algebra 126 (1989), 184-210. Zbl 0717.16029 MR 1023294

[22] E. Hairer and G. Wanner, On the Butcher group and general multi-value methods. Computing 13 (1974), 1-15. Zbl 0293.65050 MR 0403225

[23] E. Hairer, C. Lubich, and G. Wanner, Geometric numerical integration. Springer Ser. Comput. Math. 31, Springer-Verlag, Berlin 2002. Zbl 0994.65135 MR 1904823

[24] F. Hivert, J.-Ch. Novelli, and J-Y.Thibon, Trees, functional equations, and combinatorial Hopf algebras. European J. Combin. 29 (2008), 1682-1695. Zbl 1227.05272 MR 2431759

[25] M. Hoffman, Combinatorics of rooted trees and Hopf algebras. Trans. Amer. Math. Soc. 355 (2003), 3795-3811. Zbl 1048.16023 MR 1990174

[26] R. Holtkamp, Comparison of Hopf algebras on trees. Arch. Math. (Basel) 80 (2003), 368-383. Zbl 1056.16030 MR 1982837

[27] R. Holtkamp, Rooted trees appearing in products and co-products. In Combinatorics and physics, Contemp. Math. 539, Amer. Math. Soc., Providence, RI, 2011, 153-169. Zbl 1222.16026 MR 2790307 
[28] A. Iserles, Expansions that grow on trees. Notices Amer. Math. Soc. 49 (2002), 430-440. Zbl 1126.34310 MR 1892640

[29] S. A. Joni and G.-C. Rota, Coalgebras and bialgebras in combinatorics. Stud. Appl. Math. 61 (1979), 93-139. Zbl 0471.05020 MR 0544721

[30] D. E. Knuth, The art of computer programming. Volume 1: Fundamental algorithms, Addison-Wesley, Reading, Mass., 1968. Zbl 0191.17903 MR 0286317

[31] M. Livernet, A rigidity theorem for pre-Lie algebras. J. Pure Appl. Alg. 207 (2006), 1-18. Zbl 1134.17001 MR 2244257

[32] J.-L. Loday and M. Ronco, Combinatorial Hopf algebras. In Quanta of maths, Clay Math. Proc. 11, Amer. Math. Soc., Providence, RI, 2010, 347-383. Zbl 1217.16033 MR 2732058

[33] D. Manchon, Hopf algebras in renormalisation. In Handbook of algebra, Vol. 5, Elsevier/North-Holland, Amsterdam 2008, 365-427. Zbl 1215.81071 MR 2523455

[34] J.-F. Marckert, The rotation correspondence is asymptotically a dilatation. Random Structures Algorithms 24 (2004), 118-132. Zbl 1034.05016 MR 2035871

[35] J. McCammond and J. Meier, The hypertree poset and the $l^{2}$-Betti numbers of the motion group of the trivial link. Math. Ann. 328 (2004), 633-652. Zbl 1133.20040 MR 2047644

[36] H. Munthe-Kaas and W. Wright, On the Hopf algebraic structure of Lie group integrators. Found. Comput. Math. 8 (2008), 227-257. Zbl 1147.16028 MR 2407032

[37] A. Murua, The Hopf algebra of rooted trees, free Lie algebras, and Lie series. Found. Comput. Math. 6 (2006), 387-426. Zbl 1116.17004 MR 2271214

[38] W. R. Schmitt, Incidence Hopf algebras. J. Pure Appl. Algebra 96 (1994), 299-330. Zbl 0808.05101 MR 1303288

[39] D. Segal, Free left-symmetric algebras and an analogue of the Poincaré-Birkhoff-Witt theorem. J. Algebra 164 (1994), 750-772. Zbl 0831.17001 MR 1272113

[40] M. E. Sweedler, Hopf algebras. W. A. Benjamin, New York 1969. Zbl 0194.32901 MR 0252485

[41] W. Zhao, A noncommutative symmetric system over the Grossman-Larson Hopf algebra of labeled rooted trees. J. Algebraic Combin. 28 (2008), 235-260. Zbl 1152.05057 MR 2430303

[42] G. W. Zinbiel, Encyclopedia of types of algebras 2010. In Operads and universal algebra, Nankai Ser. Pure Appl. Math. Theoret. Phys. 9, World Sci. Publ., Singapore 2012, 217-297. MR 3013090

Received April 23, 2012

K. Ebrahimi-Fard, Instituto de Ciencias Matemáticas, Nicolás Cabrera, 13-15, Campus de Cantoblanco, UAM, 28049 Madrid, Spain; on leave from Université de Haute Alsace, 18 Rue des Frères Lumière, 68093 Mulhouse Cedex, France

E-mail: kurusch@icmat.es

D. Manchon, C.N.R.S.-UMR 6620, 24 avenue des Landais, Les Cézeaux, BP 80026, 63171 Aubière Cedex, France

E-mail: Dominique.Manchon@math.univ-bpclermont.fr 